\title{
Increased severity of dyspeptic symptoms related to mental stress is associated with sympathetic hyperactivity and enhanced endocrine response in patients with postprandial distress syndrome
}

\author{
F. DE Giorgi, G. SARnelli, C. CiRillo, I. G. SAVino, F. TURCO, G. NARdone, A. ROCCO \& R. CUOMO
}

Department of Clinical and Experimental Medicine, University of Naples Federico II, Naples, Italy

\begin{abstract}
Background Mental stress (MS) may alter gastric sensory-motor function. The aim of the study was to assess postprandial autonomic nervous system activity and stress hormones in response to acute mental stress in dyspeptic patients. Methods A total of 25 patients with postprandial distress syndrome (PDS; $11 \mathrm{~mol} \mathrm{~L}^{-1}$, age $35.9 \pm 9.3$ years) and 12 healthy controls $\left(5 \mathrm{~mol} \mathrm{~L}^{-1}\right.$, age $25.8 \pm 4.6$ years) underwent electrogastrography and ${ }^{13} \mathrm{C}$-octanoate gastric emptying study using a $480 \mathrm{kcal}$ solid meal. Heart rate variability (LF/HF ratio) and corticotrophin-releasing factor, adrenocorticotropic hormone (ACTH), and cortisol serum levels were also evaluated. Dyspeptic symptoms were scored by analogue visual scale and expressed as symptoms total score (TS). The protocol was repeated twice in each subject, with and without a mental stress test before the meal. Key Results Mental stress significantly increased postprandial symptoms severity in patients (TS: stress $111 \pm 18$ vs basal $50 \pm 10 ; \quad P<0.05)$. Low-/highfrequency component ratio was significantly higher in patients after MS at $120 \mathrm{~min}$ (stress $5.46 \pm 0.41 \mathrm{vS}$ basal $3.41 \pm 0.64 ; \quad P<0.01$ ) and $180 \mathrm{~min}$ (stress $5.29 \pm 0.2$ vs basal $3.58 \pm 0.19 ; P<0.05)$. During stress session, in patients we found a significantly higher ACTH level than baseline at 30, 60, 90, 150, 210, 240, and $270 \mathrm{~min}$ and a significantly higher cortisol level at 30, 60, 90, 120, 210, and $270 \mathrm{~min}$. Gastric emptying

Address for Correspondence

Rosario Cuomo, MD, Gastroenterology Unit, Department of Clinical and Experimental Medicine, Università degli Studi di Napoli 'Federico II,' Via Sergio Pansini, 5, 80131 Naples, Italy. Tel: +39 081 7463892; fax +39081 7463892;

e-mail: rcuomo@unina.it

Received: 23 March 2012

Accepted for publication: 25 July 2012
\end{abstract}

rate and electrical activity were not influenced by MS. Conclusions \& Inferences In PDS patients, administration of MS before meal increases symptoms severity by inducing sympathetic hyperactivity and increased stress hormones levels. As the gastric emptying looks not altered, we conclude that these neurohormonal responses mainly affect sensitive function.

Keywords HPA axis, LF/HF ratio, mental stress, postprandial distress syndrome.

\section{INTRODUCTION}

Functional dyspepsia (FD) is a highly prevalent gastrointestinal disorder characterized by symptoms originating from the gastroduodenal region, in the absence of underlying organic diseases that would readily explain the symptoms. The Rome III committee proposed a pathophysiologically and clinically relevant distinction between meal-induced and meal-unrelated symptoms. On this basis, FD is now subdivided into two diagnostic categories: (i) postprandial distress syndrome (PDS), characterized by postprandial fullness and early satiation, and (ii) epigastric pain syndrome (EPS), characterized by epigastric pain and burning. ${ }^{1}$ The pathogenesis of FD remains unclear: dyspeptic symptoms have been attributed to abnormal gastric motility or to visceral hypersensitivity, although psychosocial factors are also believed to play an important role. Studies concerning the relationship between stressful life events and FD have yielded conflicting results. Some studies have shown that subjects with FD experienced more stressful life events than healthy controls, $^{2-4}$ whereas other studies failed to find any significant differences. ${ }^{5}$ In FD, the comorbidity with psychiatric disorders, especially anxiety disorders, is highly frequent. ${ }^{6-9}$ It is still unclear whether these 
psychopathological factors determine health care-seeking behavior or whether they play a key role in the pathophysiology of the dyspepsia symptom complex, although there is growing evidence supporting the second hypothesis. ${ }^{3,9-11}$

How these psychosocial factors can ultimately influence FD symptoms can be explained by the well-known effects of stress on gut function. Indeed, 'stress' is a condition shared by various psychosocial factors associated with FD. Various acute stressors induces delayed gastric emptying in both experimental animals and healthy humans ${ }^{12-15}$ and, in recent times, the biological substrates of the stress response have been recognized. In brief, stressors employ subcortical circuits that induce the hypothalamic effector neurons to release corticotrophin-releasing factor $(\mathrm{CRF})$, considered the most important mediator of the central stress response. Corticotrophin-releasing factor is the primary neurohormone involved in the hallmark response to stress - i.e., the activation of hypothalamic-pituitary-adrenal (HPA) axis - but also acts as a neurotransmitter to coordinate the behavioral, autonomic, and visceral efferent limbs of the stress response. ${ }^{12,16,17}$ Components of the CRF signaling system are expressed in the brain nuclei influencing autonomic outflow to viscera; peripherally, CRF receptors are involved in the modulation of gastric myenteric activity, which influences gastric function during stress. ${ }^{18}$

In a number of experimental conditions, mental stress was able to reduce antro-duodenal motility in healthy controls but not in dyspeptic patients, suggesting that FD may arise from the effect of stress on upper gut motility in susceptible individuals. ${ }^{19,20}$ The autonomic nervous system has been proposed as the mediating mechanism for this effect. Specifically, the autonomic dysfunction found in FD patients (i.e., low vagal tone) may represent a mediating mechanism for the causal effects of personality on gastric function and the related FD symptoms. ${ }^{21}$

However, to date, a comprehensive overview of the complex brain-gut relationship in FD is still lacking. The aim of this study was to thoroughly investigate every step of this intricate link by evaluating the serum concentrations of stress hormones, the autonomic activity of the nervous system (ANS), and the gastric sensorimotor function, in response to an experimentally induced acute mental stress preceding the meal in patients with PDS. The study is designed to test our hypothesis that, in this subset of patients, the modulation of gastric function under stressful conditions is related to HPA axis and ANS activity.

\section{MATERIALS AND METHODS}

\section{Subjects}

We studied 25 patients (11 men and 14 women with a mean age of $35.9 \pm 9.3$ years) suffering from recurrent mid-upper abdominal discomfort characterized by at least two of the following symptoms: bothersome postprandial fullness, early satiety, and/or bloating. As they had been experiencing these symptoms for at least 6 months before diagnosis, the symptoms pattern was compatible with PDS as defined by the Rome III Committee. ${ }^{1}$ We also studied 12 healthy volunteers $(5$ men and 7 women with a mean age of $25.8 \pm 4.6$ years), with no history of dyspeptic symptoms, as control group.

The patients were not taking any medications known to affect gastrointestinal-motor function, cardiovascular function, or autonomic function. No patient had an evidence of organic gastroduodenal disease and a history of gastrectomy, collagen vascular disease, diabetes mellitus, or autonomic neuropathy. All female subjects underwent the experiment in the same phase of menstrual cycle, in a time period ranging from the end of menstruation to 10 days after.

All participants were screened for current symptoms of psychiatric illness using a set of validated and easy to use selfreport questionnaires. To evaluate depression, we used the Patient Health Questionnaire-9 (PHQ-9). ${ }^{22}$ Presence and severity of anxiety were tested by the Generalized Anxiety Disorder scale (GAD-7). ${ }^{23}$ To evaluate the presence of early adverse life events, we used the Early Trauma Inventory Self-Report in its short form, a brief and self-administered questionnaire evaluating the psychological impact of traumas and physical or emotional abuses in early life. ${ }^{24}$ Patients with pathologic anxiety, depression, and/or with a history of early adverse life events were excluded.

\section{Study protocol}

After an overnight fast, all subjects underwent simultaneous multichannel electrogastrography to evaluate gastric myoelectrical activity (GMA) and ${ }^{13} \mathrm{C}$-octanoic acid breath test, to study gastric emptying using a standard solid test meal. The electrogastrogram (EGG) recording was performed for $2 \mathrm{~h}, 60 \mathrm{~min}$ in the fasting state and $60 \mathrm{~min}$ postprandially. Tests started in the morning, approximately around 9:00 A.M., subjects were in supine position, in a quiet room, and were instructed to minimize their movements during the entire recording period. The test meal, composed of $480 \mathrm{kcal}$, was also used for the ${ }^{13} \mathrm{C}$-octanoic breath test.

At the same time, participants were also monitored by electrocardiogram(ECG)-Holter (Del Mar Reynolds Medical, Hertford, UK) for heart rate variability (HRV) analysis, and serum samples for CRF, adrenocorticotropic hormone (ACTH), and cortisol measurement were collected at baseline and every $30 \mathrm{~min}$ for $5 \mathrm{~h}$. Postprandial fullness and early satiety were scored every $60 \mathrm{~min}$ by visual analogue scale (VAS).

Before the study, each subject was informed that the test would start at 9:00 in the morning and that the meal would be served around 10:00 A.M. We met strictly this time schedule to avoid the effect of 'food anticipation without subsequent food reward' on cortisol serum levels, as described by Ott et al. ${ }^{25}$

The study protocol, with and without the performance of a standardized mental stress test $30 \mathrm{~min}$ before the meal, was repeated in a random order on two different days for each subject (Fig. 1). Prior to the study, all subjects were informed that they 


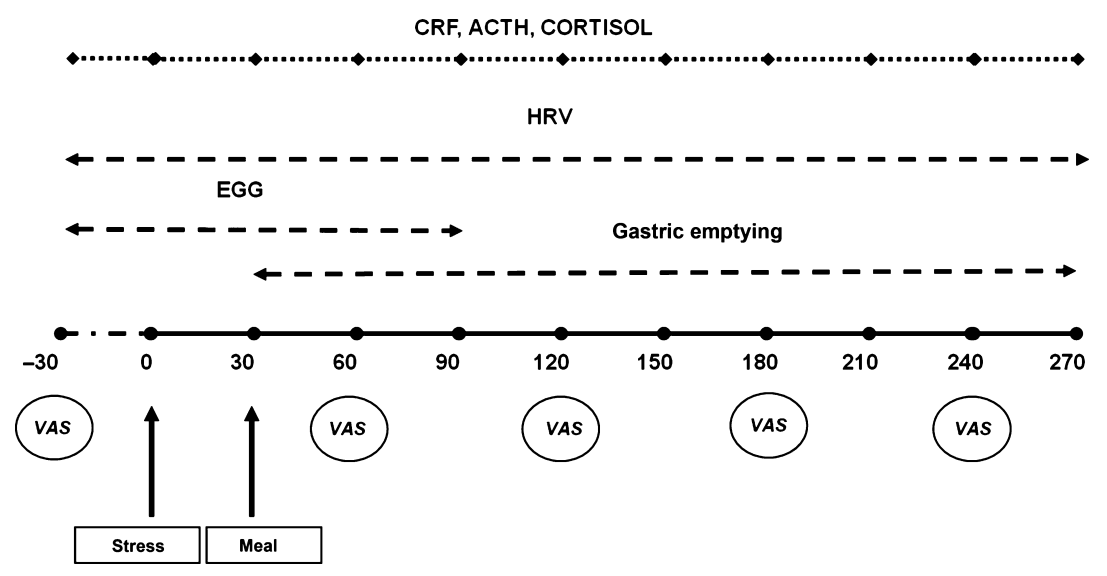

Figure 1 Schematic representation of the study protocol. After acclimatization period (T-30), mental stress, and meal were administered at T0 and T30, respectively. Gastric emptying study, by 13C-octanoate breath test, started immediately after meal and prolonged for $5 \mathrm{~h}$. Cutaneous electrogastrography was conducted for $60 \mathrm{~min}$ before and after meal. ECG Holter recording for heart rate variability analysis was performed along the whole duration of the study. Serum samples for CRF, ACTH, and cortisol detection were collected every $30 \mathrm{~min}$ for $5 \mathrm{~h}$. Symptoms severity was scored at baseline and at 60-min intervals by visual analogue scale.

would undergo two study sessions, but were unaware that one of these included a mental stress test.

This study was approved by the Ethics Committee of the 'Federico $\mathrm{II}^{\prime}$ University of Naples and was conducted in accordance with the Declaration of Helsinki for scientific research in humans. A signed written informed consent was obtained from each subject before participation.

\section{Mental stress test}

Thirty minutes before the administration of the meal, all subjects underwent a standardized mental stress test, performed as follows: after memorizing six digit numbers, they repeated these numbers in reverse for $5 \mathrm{~min}$, and performed serial subtraction of 17 from 1000 for $5 \mathrm{~min}$, under the incessant pressure of the examiner. ${ }^{26}$ Obviously, the outcome was regardless of the accuracy of the answers, as the only purpose was to provide a stressful mental engagement.

The mental stress test was performed in only one session of the study and in a random temporal order for each subject but, ultimately, the order of stress/no-stress visits was counterbalanced in both groups.

\section{Autonomic assessment by HRV analysis}

To evaluate cardiovascular autonomic function, we tested HRV by using a 3 leads ECG-Holter recorder (Aria ${ }^{\circledR}$; Del Mar Reynolds Medical). Subjects were studied in a quiet room maintained at a comfortable temperature $\left(22-24^{\circ} \mathrm{C}\right)$ in the supine position after breathing smoothly for $15 \mathrm{~min}$ to achieve acclimatization and then ECG recording was started and carried on for $5 \mathrm{~h}$. Heart rate variability data were derived from the ECG by measuring the beat-to-beat variation of the $\mathrm{R}-\mathrm{R}$ interval. The $\mathrm{R}-\mathrm{R}$ intervals were calculated, resampled, and interpolated using the Impresario ${ }^{\circledR}$ computer software (Del Mar Reynolds Medical). Power spectral analysis of HRV was then performed with fast Fourier transformation and the power values of the high-frequency component (HF: $0.15-0.40 \mathrm{~Hz}$ ), the low-frequency component (LF: $0.04-0.15 \mathrm{~Hz}$ ), and the LF/HF ratio were calculated by the software.
The LF component represents the sympathetic activity, whereas the vagal efferent activity is considered to be the major contributor to the HF component. ${ }^{27-29}$ In addition, the LF/HF ratio is considered to reflect the sympatho-vagal balance. We divided the entire recording period into 60-min intervals and calculated these three parameters for each time segment.

\section{CRF, ACTH, and cortisol assay}

To measure serum levels of CRF, ACTH, and cortisol, blood samples were collected in heparin-coated tubes at baseline and every $30 \mathrm{~min}$ for $5 \mathrm{~h}$. Blood samples were centrifuged at $4000 \times \mathbf{g}$ for $10 \mathrm{~min}$ to separate serum. The levels of CRF, ACTH, and cortisol in serum were measured using commercial ELISA kits (CRF: Phoenix Pharmaceutical Inc., Burlingame, CA, USA; ACTH: Diagnostic Biochem Canada Inc., London, ON, Canada; Cortisol: DRG International Inc., Mountainside, NJ, USA), following the manufacturer's instructions.

\section{${ }^{13} \mathrm{C}$-octanoate breath test for gastric emptying study}

All subjects were asked to consume a test meal consisting of $60 \mathrm{~g}$ white bread, $10 \mathrm{~g}$ butter, $50 \mathrm{~g}$ ham, an omelet made from one egg with egg yolk doped with $74 \mathrm{kBq}{ }^{13} \mathrm{C}$-octanoic acid (Eurisotop, Saint Aubin, France) and $100 \mathrm{~mL}$ water. The test meal contained $480 \mathrm{kcal}$ ( $19 \%$ protein, $53 \%$ carbohydrate, $31 \%$ fat). Subjects were encouraged to eat the meal within $10 \mathrm{~min}$ and were asked to strictly avoid physical activity during the study procedures. Breath samples were collected into $1.3 \mathrm{~L}$ aluminum bags before ingestion of the meal and at 15-min intervals for $240 \mathrm{~min}$ postprandially. ${ }^{13} \mathrm{CO}_{2}$-excretion in breath was subsequently analyzed using isotope-selective infrared spectroscopy to derive gastric emptying half-time.

\section{EGG recording}

Gastric myoelectrical activity in each subject was measured using surface electrogastrography with a specially designed multichan- 
nel device (Medtronic, Minneapolis, MN, USA). Before placing the electrodes, the abdominal surface where the electrodes were to be positioned was shaved, if hairy, and cleaned with sandy skin prep paste (NuPrep, Weaver and Company, Aurora, CO, USA) to reduce impedance. Six pregelified dedicated electrodes (Medtronic) were placed on the abdominal skin, including four active electrodes (numbered: 1-4), one reference electrode, and a ground electrode. Electrode 3 was placed at the mid-point between the xiphoid process and the umbilicus; electrode 4 was in the same horizontal line of electrode 3 but $4 \mathrm{~cm}$ on the right, and electrodes 2 and 1 were placed at $45^{\circ}$ angles to the upper left of electrode 3 with an interval of 4-6 cm depending on the size of the subject. Connections between each of the four active electrodes and the common reference electrode derived four-channel EGG signals. The study was performed in the supine position for $60 \mathrm{~min}$ in the fasting state and for $60 \mathrm{~min}$ after the meal. The parameters considered were dominant frequency (frequency at which the EGG power spectrum has a peak power in the range $0.5-9.0 \mathrm{cpm})$, and instability coefficient of the dominant frequency (the ratio between the standard deviation and the mean of the dominant frequencies), which is an index of the gastric slow waves variation over the time.

We considered data from Channels 2 and 3 because they are located on the cutaneous projection of the gastric pacemaker. Periods analyzed were $1 \mathrm{~h}$ preprandially and postprandially, in both basal and stress condition.

\section{Sample size assessment}

A preliminary evaluation of published data variability was performed to calculate sample size. We assumed alpha level at 0.05 and statistical power at $80 \%$. Based on the published data on ACTH, cortisol, and LF/HF ratio, we extrapolated sample sizes for patients $(12,14$, and 15 patients, according to published data for each parameter, respectively) and for controls $(9,10$, and 12 subjects, respectively). ${ }^{30-32}$

\section{Statistical analysis}

Dyspeptic symptoms severity was scored at baseline and every $60 \mathrm{~min}$ by VAS $(\mathrm{mm})$, and expressed as total score (sum of the severity of meal-related symptoms) and individual scores. Comparison between symptoms total score at baseline and during stress session was performed by ANOva for repeated measures with Bonferroni post-test for selected pairs of data. Anxiety and depression scores differences between groups were analyzed by unpaired $t$-test.

Low-, high-frequency component, and LF/HF ratio values for each 60-min interval in both study groups were compared between basal and stress condition and between controls and patients by ANOva for repeated measures with Bonferroni post-test for selected pairs of data. Serum levels at each study time, area under the curve (AUC), and time to peak of CRF, ACTH, and cortisol in basal and stress condition and between controls and patients were also compared using ANOVA for repeated measures with Bonferroni post-test for selected pairs of data. Gastric emptying time and EGG parameters were compared within each group (basal VS stress) by paired $t$-test and between the groups (controls Vs patients in basal) by unpaired $t$-test. Analysis of correlation between anxiety scores and HRV data and between HPA axis hormones and the other parameters studied was performed by Spearman's rank linear correlation. All data were expressed as mean \pm SEM. Differences were considered statistically significant when $P$ was $<0.05$.

\section{RESULTS}

\section{Dyspeptic symptoms}

In patients, total score of dyspeptic symptoms was evidently higher in the postprandial period and significantly higher when mental stress was applied, compared with basal condition, at $180 \mathrm{~min}$ (patients basal $50 \pm 10$ vs patients stress $111 \pm 18 ; P<0.05$; Fig. 2). Individual scores of meal-related symptoms showed a tendency to increase in stress condition, with satiation showing the greatest increase, although the differences were not statistically significant (data not shown). Controls, by definition, had no dyspeptic symptoms in either basal or stress condition.

\section{Anxiety and depression scores}

Patients showed a higher anxiety score than controls (patients $9.8 \pm 4.6$ VS controls $3.5 \pm 2.07 ; P<0.05$ ). According to the GAD-7 scoring method, this accounts for a mild-to-moderate anxiety grade in patients. Instead, no differences between groups were observed at the depression score (Table 1).

\section{HRV}

A significant increase in the LF component power, indicating sympathetic activity, was observed in patients during stress condition at $120 \mathrm{~min}$ (stress $77.79 \mathrm{msec}^{2} \mathrm{~Hz}^{-1} \pm 10.23$ vs basal $69.26 \mathrm{msec}^{2} \mathrm{~Hz}^{-1} \pm$

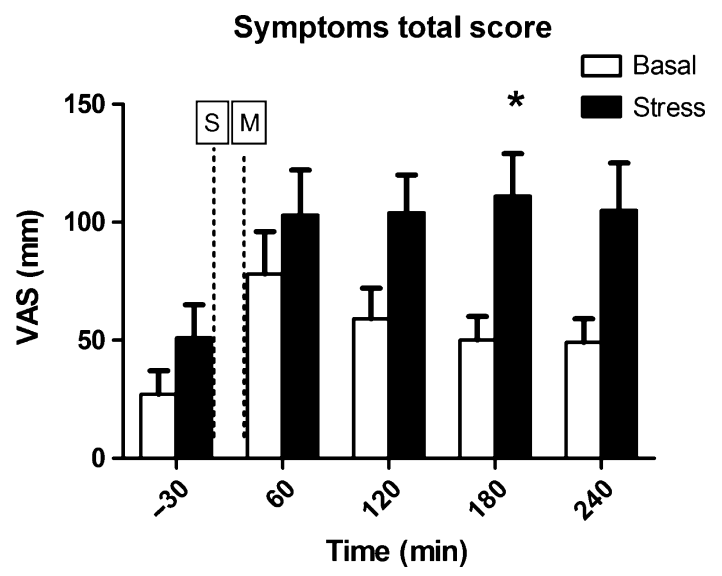

Figure 2 Symptoms severity in patients, in basal and stress condition. In this graph, symptoms severity is expressed as total score (sum of visual analogue scale values of postprandial fullness and satiation). Patients tend to be more symptomatic in stress condition, with a significant difference at 150 min after meal (T180). In stress condition, a trend to symptoms increase over the time is also noticeable, whereas in basal condition there is an opposite behavior. S, stress; M, meal. ${ }^{\star} P<0.05$. 
Table 1 Anxiety and depression scores of patients and controls, assessed, respectively, by GAD-7 and PHQ-9

\begin{tabular}{llr}
\hline Patients & $9.8 \pm 4.6^{\star}$ & $5.8 \pm 2.8$ \\
Controls & $3.5 \pm 2.1$ & $3.87 \pm 3.3$
\end{tabular}

GAD-7, Generalized Anxiety Scale; PHQ-9, Patient Health Questionnaire; PDS, postprandial distress syndrome.

Patients with PDS showed significantly higher anxiety scores than controls in the 2 weeks preceding the experiment. Depression scores did not show any difference between the two groups. ${ }^{\star} P<0.05$ vs Controls.

$11.55 \mathrm{Vs} ; P=0.01$ ) and $180 \mathrm{~min}$ (stress $77.86 \mathrm{msec}^{2}$ $\mathrm{Hz}^{-1} \pm 2.46$ vs basal $\left.71.65 \mathrm{msec}^{2} \mathrm{~Hz}^{-1} \pm 2.34 ; P<0.05\right)$. Conversely, the HF component power, reflecting vagal activity, was significantly decreased at the same time point during stress: $120 \mathrm{~min}$ (basal $26.22 \mathrm{msec}^{2} \mathrm{~Hz}^{-1} \pm$ 5.94 Vs stress $\left.18.56 \mathrm{msec}^{2} \mathrm{~Hz}^{-1} \pm 2.1 ; P<0.05\right)$ and $180 \mathrm{~min}$ (stress $19.04 \mathrm{msec}^{2} \mathrm{~Hz}^{-1} \pm 2.24$ vs basal $\left.24.66 \mathrm{msec}^{2} \mathrm{~Hz}^{-1} \pm 5.96 ; P<0.05\right)$.

Consequently, LF/HF ratio was significantly higher in patients during stress condition, compared with baseline at $120 \mathrm{~min}$ (stress $5.46 \pm 0.41 \mathrm{vs}$ basal $3.41 \pm 0.64$; $P<0.01$ ) and $180 \mathrm{~min}$ (stress $5.29 \pm 0.2$ vs basal $3.58 \pm 0.19 ; P<0.05)$, thus indicating a postprandial predominance of the sympathetic output induced by stress (Fig. 3). In controls, we found no difference in HRV parameters between basal and stress conditions.

We have corrected individuals LF/HF ratio values for the anxiety scores in each subject and performed a comparison of corrected values between basal and stress conditions, without finding any statistical significance. We have also performed a correlation between anxiety scores and LF/HF ratio at each time point, and again found no significance.

\section{Stress hormones}

Serum levels of CRF, ACTH, and cortisol were measured every $30 \mathrm{~min}$ in both study conditions and compared at each time point in patients and controls. No differences were found for CRF basal values between the groups, whereas ACTH and cortisol basal values were significantly lower $(P<0.05)$ in patients with respect to controls (ACTH: -30, 0, 30, 60, and $240 \mathrm{~min}$; cortisol: $0,30,60,150,180$, and $270 \mathrm{~min})$. In patients, during the stress condition, we found a significantly higher ACTH level than baseline at 30,60, 90, 150, 210, 240, and 270 min (Fig. 4). During stress session, we found also a significantly higher cortisol level than baseline in patients at 30, 60, 90, 120, 210, and $270 \mathrm{~min}$ (Fig. 5). Corticotrophin-releasing factor levels instead showed no difference between basal and stress condition in patients. In controls, no differences were observed in hormone levels between the two study sessions.

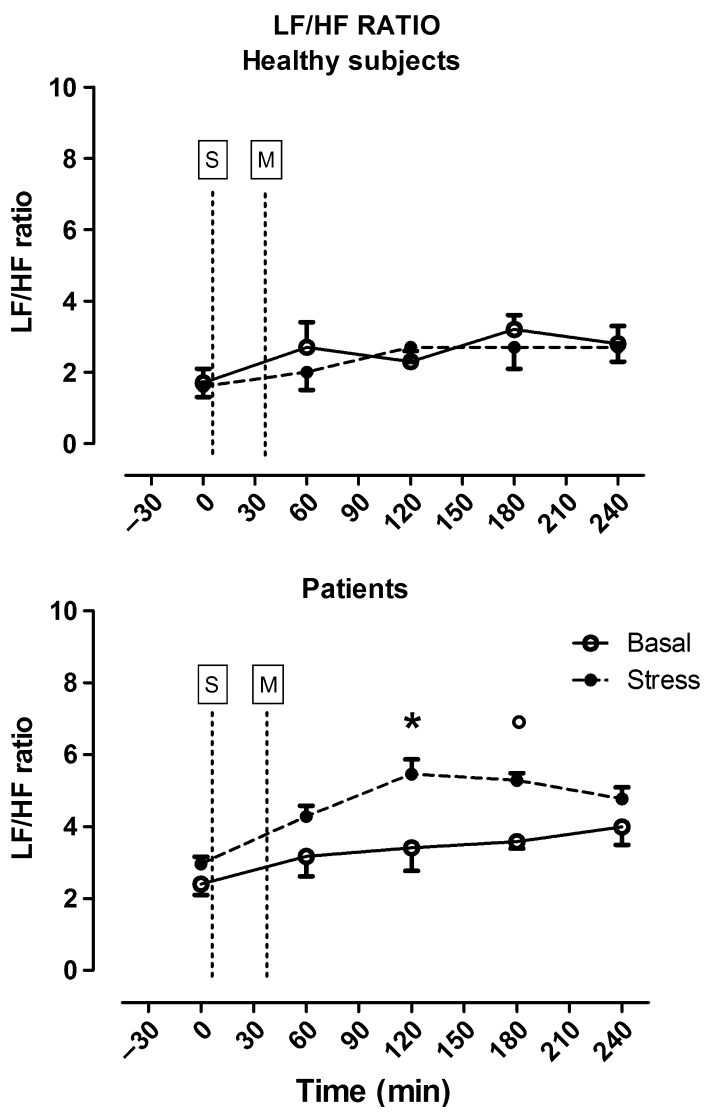

Figure 3 Low-/high-frequency component (LF/HF) ratio in patients and controls during basal and stress sessions of this study. In patients, mental stress induces a prolonged increase of LF/HF ratio in the postprandial period, interpreted as an increase in sympathetic output. In controls, no differences were found between basal and stress condition. Each plotted value on the graph expresses the LF/HF ratio of the previous $60 \mathrm{~min}$. S, stress; $\mathrm{M}$, meal. ${ }^{\star} P<0.01$ and ${ }^{\circ} \mathrm{P}<0.05$ vs basal.

In addition, we failed to find significant differences in AUC and time to peak of CRF, ACTH, and cortisol between basal and stress condition in either group, and even comparing patients and controls in both conditions.

To confirm the role of HPA axis hormones in the modulation of gastrointestinal visceral response to mental stress, we have also performed a correlation between CRF, ACTH, cortisol AUCs, and some of the other parameters evaluated (LF/HF ratio, symptoms score) at each time point: a significant correlation was found in patients between AUC of ACTH and symptoms total score (VAS) at $150 \mathrm{~min}(r=0.70 ; P<0.01)$.

\section{Gastric emptying time}

In basal condition, patients showed a significantly higher gastric emptying half-time than controls, (patients $159.4 \mathrm{~min} \pm 21.8$ vs controls $81.58 \mathrm{~min} \pm$ 5.35; $P<0.05)$; however, the comparison between basal 

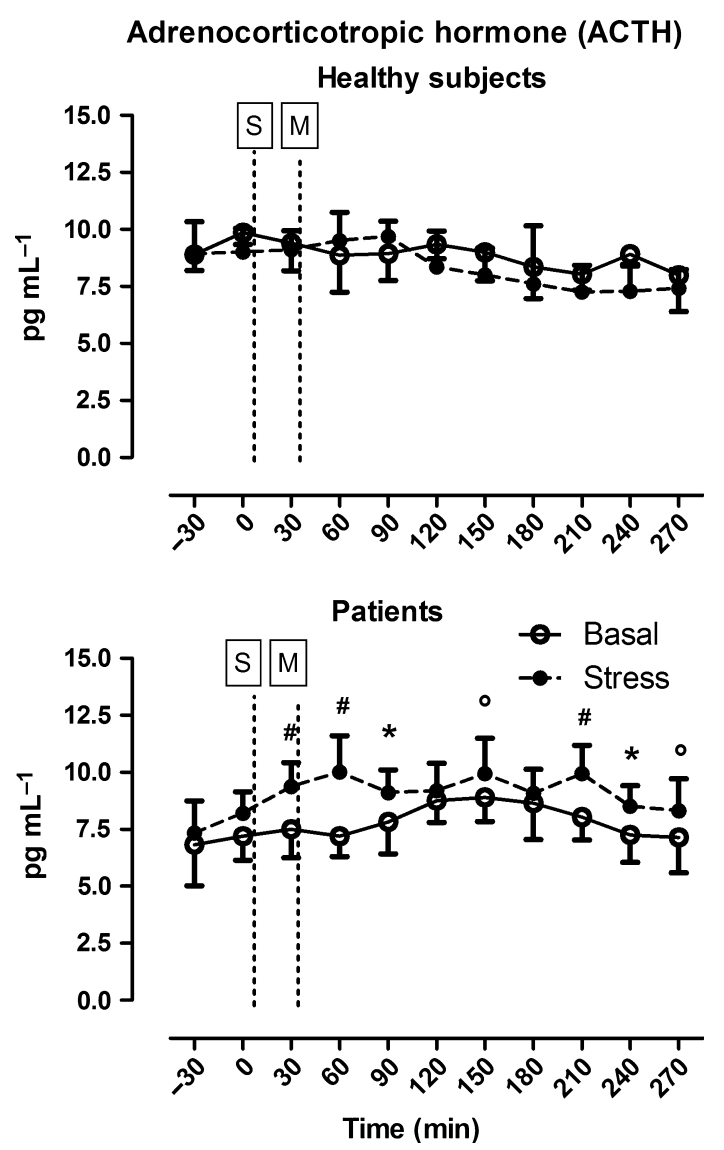

Figure 4 Adrenocorticotropic hormone (ACTH) serum levels in healthy subjects and patients. In patients, a significantly higher ACTH level was found in stress condition, whereas in healthy subjects no modifications were recorded. S, stress; $\mathrm{M}$, meal. ${ }^{\#} P<0.001,{ }^{\star} P<0.01$, and ${ }^{\circ} \mathrm{P}<0.05$ vs basal.

and stress condition in either patients and controls did not show any difference (Fig. 6).

\section{EGG data}

Dominant frequency and instability coefficient of the selected periods ( $1 \mathrm{~h}$ preprandial and postprandial) did not show any modification between basal and stress condition in either study group, nor did we find any difference comparing EGG data of patients and controls. In a further analysis of EGG data in the fasting state, these parameters did not show any significant difference between $30 \mathrm{~min}$ before and after the administration of mental stress in both study groups (Table 2).

\section{DISCUSSION}

To our knowledge, this study is the first attempt to fully characterize the neuroendocrine pathways involved in the gastric response to mental stress in

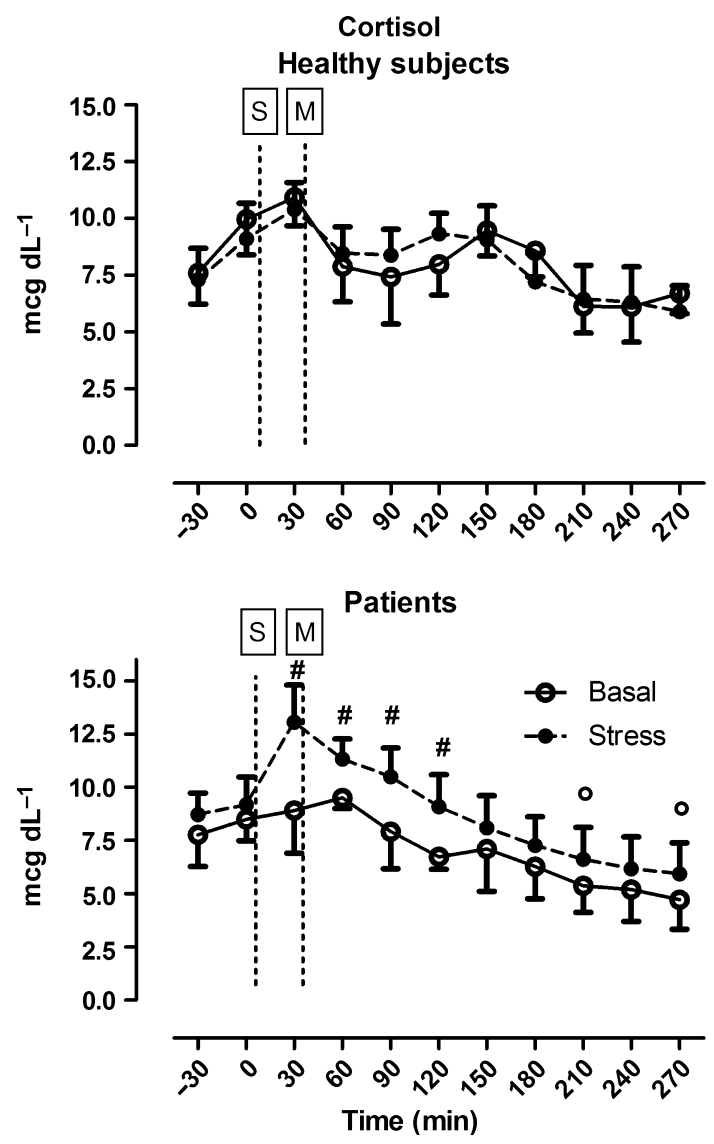

Figure 5 Cortisol serum levels in healthy subjects and patients. Patients showed a significantly higher level in stress condition, whereas in healthy subjects cortisol levels in both conditions were similar. S, stress; M, meal. ${ }^{\#} P<0.001$ and ${ }^{\circ} P<0.05$ vs basal.

patients with PDS. We found that the increased severity of dyspeptic symptoms induced by acute mental stress in patients with PDS is associated with an enhanced sympathetic output and higher serum levels of stress hormones, ACTH, and cortisol.

Psychological stress is widely believed to play a major role in functional gastrointestinal disorders, especially irritable bowel syndrome (IBS) and dyspepsia, by precipitating exacerbation of symptoms, although the pathophysiology of this phenomenon is not well recognized. Previous data clearly show that association between FD and psychopathology could not be explained only by a tendency to a greater health care seeking. Castillo et al. ${ }^{33}$ reported higher scores of somatization in dyspeptic patients than in non-dyspeptic controls and, interestingly, dyspeptic patients did not differ from controls in gastric emptying and accommodation, suggesting the importance of somatization over the impairment of gastric motor function in the generation and perception of dyspeptic symptoms. Moreover, van Oudenhove et al. ${ }^{34}$ reported 


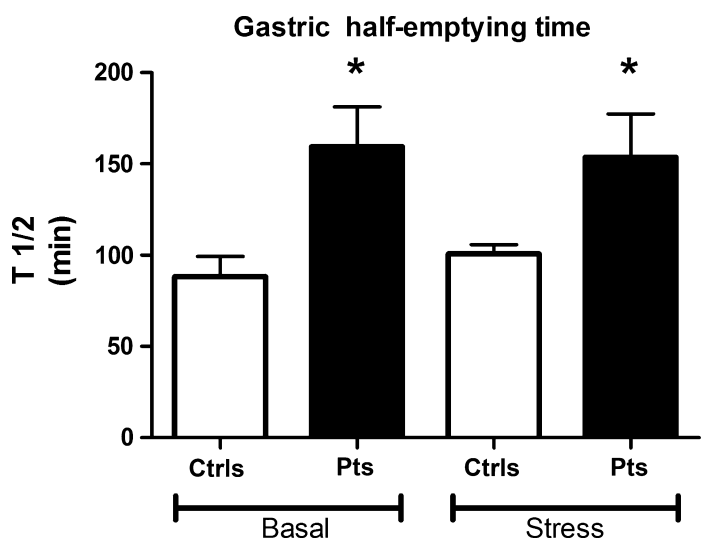

Figure 6 Gastric half-emptying time in healthy subjects and patients. Patients showed higher gastric emptying time than controls $\left({ }^{\star} P<0.05\right)$, but mental stress did not influence gastric emptying rate both in patients and controls.

Table 2 Electrogastrographic parameters in patients and controls in basal and stress conditions, recorded pre- and postprandially at Channel 3

\begin{tabular}{llllll}
\hline \multicolumn{3}{c}{ Basal } & & \multicolumn{1}{l}{ Stress } & \\
\cline { 2 - 3 } \cline { 5 - 6 } \cline { 5 - 6 } & Preprandial & Postprandial & & Preprandial & Postprandial \\
\hline Dominant frequency & & & & \\
Patients & $2.80 \pm 0.08$ & $2.90 \pm 0.06$ & & $2.80 \pm 0.07$ & $2.90 \pm 0.10$ \\
Controls & $2.70 \pm 0.15$ & $2.80 \pm 0.15$ & & $2.70 \pm 0.23$ & $3.00 \pm 0.07$ \\
Instability coefficient & & & & \\
Patients & $0.10 \pm 0.03$ & $0.09 \pm 0.02$ & & $0.09 \pm 0.04$ & $0.08 \pm 0.01$ \\
Controls & $0.20 \pm 0.13$ & $0.07 \pm 0.01$ & & $0.30 \pm 0.09$ & $0.15 \pm 0.07$ \\
\hline
\end{tabular}

DF, dominant frequency; IC, instability coefficient; EGG, electrogastrogram.

Variables considered are DF, defined as the frequency at which the EGG power spectrum has a peak power in the range: $0.5-9.0 \mathrm{cpm}$, and IC, namely the ratio between the standard deviation and the mean of the dominant frequencies. Both parameters did not show any significant difference between patients and controls, and in each group, any modification between basal and stress condition.

that somatization was the most important factor for symptom severity and weight loss in FD. Therefore, psychopathological factors may be closely associated with the pathophysiology of FD, at least in a subset of patients. Furthermore, emotional stressors may cause disturbances at every level of the brain-gut axis, including the central, autonomic, and enteric nervous system, affecting the regulation of visceral perception and thus inducing a state of visceral hypersensitivity ${ }^{35}$; this would explain the increased severity of postprandial dyspeptic symptoms in our PDS patients after the administration of mental stress.

We chose to enroll pure PDS patients because it has been demonstrated that the diagnosis of PDS, but not EPS, is independently associated with psychopathological factors, specifically in the dimension of somatiza- tion, depression, and phobia. ${ }^{36}$ Differently from EPS, PDS was also shown to be linked to major anxiety in a population-based study. ${ }^{37}$ For these reasons, PDS patients likely represent a subgroup where the influence of mental stress on gastric pathophysiology could be much more evident. Actually, analysis by the GAD7 questionnaire, a validated and efficient tool for screening and scoring of $\mathrm{GAD}^{23}$ showed higher scores of anxiety in our PDS patients compared with controls, confirming previous data and suggesting a greater susceptibility to psychological stress for PDS patients.

In this study, we proposed to investigate the potential mechanisms mediating the link between psychiatric disorders, mental stress, and gastric sensorimotor function. These mechanisms are to be identified mainly in the autonomic nervous system and the stress-hormone system provided by the HPA axis. Consistently with previous evidence, we found an enhanced sympathetic activity and concurrent low vagal tone after the administration of acute mental stress and solid meal in PDS patients. Autonomic nervous system abnormalities have been reported in adults with FD. The most common finding has been decreased vagal tone - although this was not constant across all studies. ${ }^{20,21,38,39}$ Chen et al. ${ }^{40}$ found an inhibition of postprandial vagal activity and an increase in postprandial sympathetic activity following mental stress, along with the inhibition of GMA. Tougas et al. ${ }^{31}$ reported basal sympathetic hyperactivity followed by increased vagal output during esophageal acid infusion in patients who experienced anginalike chest pain during the infusion ('acid sensitive' patients), which was not seen in those without symptoms: the authors concluded that increased basal sympathetic activity was an underlying neural abnormality associated with esophageal hypersensitivity to acid exposure, possibly consequent to longstanding psychophysical factors associated with anxiety and stress. Similarly, in a previous study, we identified a subgroup of patients with gastroesophageal reflux disease and cardiac arrhythmias in whom the esophageal acid stimulation elicited cardiac autonomic reflexes: all these patients had basal sympathetic hyperactivity over $24 \mathrm{~h}$, associated with increased anxiety levels. ${ }^{32}$

Data from HRV analysis in our PDS patients clearly show a prolonged postprandial increase in sympathetic output solely in the session of the study where acute mental stress precedes the meal, but this effect does not seem to affect either GMA or gastric emptying time. Therefore, we can assume that PDS patients have an underlying hyperactivity of the sympathetic system, which can be enhanced by mental stress and meal, 
presumably also in relation to the higher scores of anxiety observed in the patients themselves, as anxiety is known to be associated with increased sympathetic tone. ${ }^{41,42}$ The lack of significant differences between LF/HF ratio values corrected for anxiety score between basal and stress conditions in patients confirms that the higher level of underlying anxiety in patients represents an important factor to determine the enhanced neuroendocrine response to mental stress.

Another crucial point to be discussed is the postprandial and stress-induced increase in HPA axis hormones observed only in PDS patients. Very few studies have investigated HPA parameters in functional gastrointestinal patients, and they have yielded inconclusive results. Heitkemper et al. ${ }^{43}$ found no significant differences in urinary cortisol between IBS patients and healthy controls, whereas other reports indicated an overactivation of the HPA axis in IBS patients. ${ }^{44,45}$ Patacchioli et al. ${ }^{46}$ found that salivary cortisol levels were higher in the morning and lower in the evening in IBS patients compared with controls. Moreover, Posserud et al. ${ }^{47}$ found higher levels of CRF and ACTH in IBS patients than in controls, after mental stress. Analyzing our results, firstly, we should underline the lower basal levels of ACTH and cortisol in our patients respect to controls. These data confirm a previous observation on patients with functional gastrointestinal disorders showing a lower adrenocortical activity compared with healthy controls. ${ }^{48}$ However, the most interesting finding of this study is the assessment of HPA axis activation induced by meal and mental stress in PDS patients, in terms of increased serum levels of ACTH and cortisol but not CRF. The reason why we failed to find an increase in CRF levels may be explained in several ways. The stress response of the HPA axis is rather complex and modulated by numerous factors. Hypothetically, one would expect that a stress-induced activation of CRF neurons in the hypothalamic paraventricular nucleus resulted in a subsequent linear release of ACTH from the pituitary and of cortisol from the adrenals gland. However, research investigating the linearity between CRF and ACTH release suggests that only part of the variance of ACTH and glucocorticoid release can be explained by CRF release under stimulated ${ }^{49}$ and unstimulated conditions. ${ }^{50}$ Most likely, the lack of evidence for increased CRF can be explained by the intrinsic difficulty to detect peripherally a very labile molecule with a short plasma half-life; it is also presumable that peripheral levels of CRF are not completely representative of central nervous system levels. Moreover, CRF action is also dependent on CRF receptor distribution and proportion of the different subtypes of receptors as there are data suggesting somewhat opposite effects when stimulated. ${ }^{16}$ Nevertheless, it has been shown that, in healthy volunteers, peripheral infusion of CRF reduces basal gastric fundic tone, but has no effect on meal-induced accommodation or visceral sensitivity to gastric distension: this finding suggests that in healthy volunteers, peripheral corticotropin-releasing hormone does not seem to be involved in the onset of dyspeptic symptoms. ${ }^{51}$ Ultimately, as the well recognized importance of CRF in stress-related visceral response has not been established by change in circulating levels but using CRF receptor antagonists or local alterations of CRF expression in tissue, ${ }^{12}$ our findings are obviously not conclusive to establish the role of CRF.

In summary, the stress-induced increase in dyspeptic symptoms observed in our PDS patients is mediated by neuroendocrine responses, which do not seem to affect gastric emptying or gastric myoelectric activity; therefore we can speculate that acute mental stress is able to modify only the gastric sensitive function. A major limitation of this study is the assessment of the gastric sensitive function only by VAS, and the lack of an objective evaluation, as that provided by barostat-based fundic distension, could make the results less reliable. However, as our goal was to evaluate the impact of mental stress on meal-related dyspeptic symptoms, we proposed to simulate a daily and usual activity like meal ingestion, rather than carrying out invasive tests.

Another potential bias of this study is that the stressinduced anxiety and increased vigilance could in itself explain the increase in dyspeptic symptoms rather than being a product of an altered neuroendocrine response.

In conclusion, our findings contribute to shed light on the complex link between the central nervous system and the gastrointestinal tract, and specifically to help to clarify the mechanisms that underlie functional gut modifications induced by mental stress in FD. The finding of an altered visceral sensitivity, induced by mental stress and mediated by neuroendocrine responses, opens the way for future investigations and identifies a target for the development of new therapeutic approaches for FD in selected patients.

\section{FUNDING}

This work was supported by research grant from the Italian Ministry of University and Research (COFIN projects NO. 2004062155 to RC).

\section{DISCLOSURE}

The authors have no competing interests to declare. 


\section{AUTHOR CONTRIBUTION}

FG contributed to the conception, experimental work, analysis and interpretation of the data, and the draft of the manuscript; GS contributed to the conception, interpretation of the data, and the draft of the manuscript; IGS, FT, GN, AR, and CC contributed to the experimental work and critical revision of the manuscript; RC contributed to the conception, interpretation of the data, draft, critical revision, and final approval of the manuscript. The authors have all read and approved the manuscript. Special thanks to Mrs. Rosanna Scala for linguistic assistance.

\section{REFERENCES}

1 Tack J, Talley NJ, Camilleri $\mathrm{M}$ et al. Functional gastroduodenal disorders. Gastroenterology 2006; 130: 1466-79.

2 Jorgensen LS, Bonlokke L, Christensen NJ. Life strain, life events, and autonomic response to a psychological stressor in patients with chronic upper abdominal pain. Scand I Gastroenterol 1986; 21: 605-13.

3 Locke GR, Weaver AL, Melton LJ et al. Psychosocial factors are linked to functional gastrointestinal disorders: a population based nested casecontrol study. Am I Gastroenterol 2004; 99: 350-7.

4 Chen TS, Lee YC, Chang FY et al. Psychosocial distress is associated with abnormal gastric myoelectrical activity in patients with functional dyspepsia. Scand I Gastroenterol 2006; 41: 791-6.

5 Hefeiz HB, Al-Quorain A, Al-Mangoor $\mathrm{S}$ et al. Life events stress in functional dyspepsia: a case-control study. Eur I Gastroenterol Hepatol 1997; 9: 21-6.

6 Budavari AI, Olden KW. Psychosocial aspects of functional gastrointestinal disorders. Gastroenterol Clin North Am 2003; 32: 477-506.

7 Wilhelmsen I, Haug TT, Ursin H et al. Discriminant analysis of factors distinguishing patients with functional dyspepsia from patients with duodenal ulcer. Significance of somatization. Dig Dis Sci 1995; 40: 1105-11.

8 Fischler B, Tack J, De Gucht V et al. Heterogeneity of symptom pattern, psychosocial factors, and pathophysiological mechanisms in severe functional dyspepsia. Gastroenterology 2003; 124: 903-10.

9 Van Oudenhove L, Demyttenaere K, Tack J et al. Central nervous system involvement in functional gastrointestinal disorders. Best Pract Res Clin Gastroenterol 2004; 18: 663-80.

10 Geeraerts B, Vanderberghe J, Van Oudenhove L et al. Influence of experimentally induced anxiety on gastric sensorimotor function in humans. Gastroenterology 2005; 129: 1437-44.

11 Van Oudenhove L, Vandenberghe J, Vos $\mathrm{R}$ et al. Abuse history, depression, and somatization are associated with gastric sensitivity and gastric emptying in functional dyspepsia. Psychosom Med 2011; 73: 648-55.

12 Taché Y, Martinez V, Million M et al. Stress and the gastrointestinal tract: III. Stress-related alterations of gut motor function: role of brain corticotropin-releasing factor receptors. Am I Physiol Gastrointest Liver Physiol 2001; 280: G173-7.

13 Stam R, Croiset G, Akkermans LM et al. Effects of novelty and conditioned fear on small intestinal and colonic and behavior in the rat. Physiol Behav 1995; 58: 803-9.

14 Rao SS, Hatfield RA, Suls JM et al. Psychological and physical stress induce differential effects on human colonic motility. Am I Gastroenterol 1998; 93: 985-90.

15 Mönnikes H, Tebbe JJ, Hildebrandt M et al. Role of stress in functional gastrointestinal disorders. Evidence for stress-induced alterations in gastrointestinal motility and sensitivity. Dig Dis 2001; 19: 201-11.

16 Bale TL, Vale WW. CRF and CRF receptors: role in stress responsivity and other behaviors. Annu Rev Pharmacol Toxicol 2004; 44: 525-57.

17 Caso JR, Leza JC, Menchen L. The effects of physical and psychological stress on the gastrointestinal tract: lessons from animal models. Curr Mol Med 2008; 8: 299-312.

18 Stengel A, Tachè Y. Neuroendocrine control of the gut during stress: corticotrophin-releasing factor signalling pathways in the spotlight. Annu Rev Physiol 2009; 71: 219-39.

19 Hveem K, Hausken T, Svebak S et al. Gastric antral motility in functional dyspepsia. Effect of mental stress and cisapride. Scand I Gastroenterol 1996; 31: 452-7.

20 Hausken T, Svebak S, Wilhelmsen I et al. Low vagal tone and antral dysmotility in patients with func- tional dyspepsia. Psychosom Med 1993; 55: 12-22.

21 Haug TT, Svebak S, Hausken T et al. Low vagal activity as mediating mechanism for the relationship between personality factors and gastric symptoms in functional dyspepsia. Psychosom Med 1994; 56: 181-6.

22 Kroenke K, Spitzer RL. The PHQ-9: a new depression and diagnostic severity measure. Psychiatr Ann 2002; 32: 509-21.

23 Spitzer RL, Kroenke K, Williams JB. et al. A brief measure for assessing generalized anxiety disorder: the GAD-7. Arch Intern Med 2006; 166: 1092-7.

24 Bremner JD, Bolus R, Mayer EA. Psychometric properties of the Early Trauma Inventory-Self Report. I Nerv Ment Dis 2007; 195: 211-8.

25 Ott V, Friedrich M, Prilop S et al. Food anticipation and subsequent food withdrawal increase serum cortisol in healthy men. Physiol Behav 2011; 103: 594-9.

26 Yoshida K, Utsunomiya T, Morooka $\mathrm{T}$ et al. Mental stress test is an effective inducer of vasospastic angina pectoris: comparison with cold pressor, hyperventilation and master two-step exercise test. Int I Cardiol 1999; 70: 155-63.

27 Kamath MW, Fallen EL. Power spectral analysis of heart rate variability: a noninvasive signature of cardiac autonomic function. Crit Rev Biomed Eng 1993; 21: 245-311.

28 Bootsma MC, Swenne C, vanBolhuis $\mathrm{H}$ et al. Heart rate and heart rate variability as indexes of sympathovagal balance. Am I Physiol 1994; 266: 1565-71.

29 Goldberger JJ. Sympathovagal balance: how should we measure it? Am J Physiol 1999; 276: 1273-80.

30 Kudielka BM, Buske-Kirschbaum A, Hellhammer DH et al. HPA axis responses to laboratory psychosocial stress in healthy elderly adults, younger adults and children: impact of age and gender. Psychoneuroendocrinology 2004; 29: 83-98. 
31 Tougas G, Spaziani R, Hollerbach S et al. Cardiac autonomic function and oesophageal acid sensitivity in patients with non-cardiac chest pain. Gut 2001; 49: 706-12.

32 Cuomo R, De Giorgi F, Adinolfi L et al. Oesophageal acid exposure and altered neurocardiac function in patients with GERD and idiopathic cardiac dysrhythmias. Aliment Pharmacol Ther 2006; 2: 361-70.

33 Castillo EJ, Camilleri M, Locke GR et al. A community-based, controlled study of the epidemiology and pathophysiology of dyspepsia. Clin Gas troenterol Hepatol 2004; 2: 985-96.

34 Van Oudenhove L, Vandenberghe I, Geeraerts B et al. Determinants of symptoms in functional dyspepsia: gastric sensorimotor function, psychosocial factors or somatisation? Gut 2008; 57: 1666-73.

35 Larauche M, Mulak A, Tachè Y. Stress-related alterations of visceral sensation: animal models for irritable bowel syndrome study. Neurogastroenterol Motil 2011; 17: 213-34.

36 Hsu YC, Liou JM, Liao SC et al. Psychopathology and personality trait in subgroups of functional dyspepsia based on Rome III criteria. Am I Gastroenterol 2009; 104: 2534-42.

37 Aro P, Talley NJ, Ronkainen J et al. Anxiety is associated with uninvestigated and functional dyspepsia (Rome III criteria) in a Swedish population-based study. Gastroenterology 2009; 137: 94-100.
38 Lorena SL, Figueiredo MJ, Almeida JR et al. Autonomic function in patients with functional dyspepsia assessed by 24-hour heart rate variability. Dig Dis Sci 2002; 47: 27-31.

39 Park DI, Rhee PL, Kim YH et al. Role of autonomic dysfunction in patients with functional dyspepsia. Dig Liver Dis 2001; 33: 464-71.

40 Yin J, Levanon D, Chen JD. Inhibitory effects of stress on postprandial gastric myoelectrical activity and vagal tone in healthy subjects. Neurogastroenterol Motil 2004; 16: 737-44.

41 Kawachi I, Sparrow D, Vokonas PS et al. Decreased heart rate variability in men with phobic anxiety (data from the Normative Aging Study). Am J Cardiol 1995; 75: 882-5.

42 Piccirillo G, Elvira S, Bucca C et al. Abnormal passive head-up tilt test in subjects with symptoms of anxiety power spectral analysis study of heart rate and blood pressure. Int $J$ Cardiol 1997; 60: 121-31.

43 Heitkemper $M$, Jarrett M, Cain K et al. Increased urine catecholamines and cortisol in women with irritable bowel syndrome. Am I Gastroenterol 1996; 91: 906-13.

44 Dinan TG, Quigley EM, Ahmed SM et al. Hypothalamic-pituitary-gut axis dysregulation in irritable bowel syndrome: plasma cytokines as a potential biomarker? Gastroenterology 2006; 130: 304-11.

45 Chang L, Sundaresh S, Elliott J et al. Dysregulation of the hypothalamic- pituitary-adrenal (HPA) axis in irritable bowel syndrome. Neurogastroenterol Motil 2009; 21: 149-59.

46 Patacchioli FR, Angelucci L, Dellerba $\mathrm{G}$ et al. Actual stress, psychopathology and salivary cortisol levels in the irritable bowel syndrome (IBS). I Endocrinol Invest 2001; 24: 173-7.

47 Posserud I, Agerforz P, Ekman R et al. Altered visceral perceptual and neuroendocrine response in patients with irritable bowel syndrome during mental stress. Gut 2004; 53: 1102-8.

48 Böhmelt AH, Nater UM, Franke S et al. Basal and stimulated hypothalamic-pituitary. Adrenal axis activity in patients with functional gastrointestinal disorders and healthy controls. Psychosom Med 2005; 67: 288-94.

49 Carlson DE, Chiu WC, Fiedler SM et al. Central neural distribution of immunoreactive Fos and $\mathrm{CRH}$ in relation to plasma ACTH and corticosterone during sepsis in the rat. Exp Neurol 2007; 205: 485-500.

50 Watts AG. Glucocorticoid regulation of peptide genes in neuroendocrine CRH neurons: a complexity beyond negative feedback. Front Neuroendocrinol 2005; 4: 109-30.

51 van den Elzen $\mathrm{BD}$, van den Wijngaard RM, Tytgat GN et al. Influence of corticotropin-releasing hormone on gastric sensitivity and motor function in healthy volunteers. Eur I Gastroenterol Hepatol 2007; 19: 401-7. 